\title{
Quasinormal modes for the SdS black hole : an analytical approximation scheme
}

\author{
V. Suneeta * \\ Theoretische Physik, Ludwig-Maximilians-Universität, \\ Theresienstrasse 37, D-80333, München, Germany
}

(Dated: October 25, 2018)

\begin{abstract}
Quasinormal modes for scalar field perturbations of a Schwarzschild-de Sitter (SdS) black hole are investigated. An analytical approximation is proposed for the problem. The quasinormal modes are evaluated for this approximate model in the limit when black hole mass is much smaller than the radius of curvature of the spacetime. The model mirrors some striking features observed in numerical studies of time behaviour of scalar perturbations of the SdS black hole. In particular, it shows the presence of two sets of modes relevant at two different time scales, proportional to the surface gravities of the black hole and cosmological horizons respectively. These quasinormal modes are not complete - another feature observed in the numerical studies. Refinements of this model to yield more accurate quantitative agreement with numerical results are discussed. Further investigations of this model are outlined, which would provide a valuable insight into time behaviour of perturbations in the SdS spacetime.
\end{abstract}

PACS numbers: 04.70.-s, 04.70.Bw, 04.30.Nk

\section{INTRODUCTION}

A characteristic feature of the response of a black hole to external perturbations is the appearance of quasinormal modes (QNMs). The presence of these modes was first noted in a study of perturbations of the Schwarzschild black hole, by Vishveshvara 1]. Since then, QNMs for asymptotically flat black holes have been computed by a variety of numerical and analytical approximation methods. A detailed review of this work can be found in [2, 3].

QNMs were first found in a stability analysis of black holes. However, the observation that these damped oscillations are intrinsic characteristics of the black hole exterior geometry and depend only on the black hole parameters implies that they are the imprint of a black hole in its response to perturbations. Further, QNMs are even seen at intermediate/late times in fully non-linear situations like systems undergoing gravitational collapse. Thus they are expected to play a significant role in the search for gravitational waves and black holes. Recent evidence for a non-zero positive cosmological constant points to the importance of studying black holes in such a background. The simplest black hole in this class is a Schwarzschild black hole in de Sitter space (SdS). The QNM spectra for gravitational perturbations of this black hole have been investigated in [4, 5] using numerical and analytical approximation techniques. QNM spectra for the SdS black hole in the near-extremal case when the two horizons are nearly coincident (i.e, the black hole mass and radius of curvature of the spacetime of the same order) are derived in 6]. An interesting numerical study of evolution of scalar fields in the SdS spacetime has been performed in [7, 8]. This numerical study reveals the differences between the response of the Schwarzschild black hole and the SdS black hole to perturbations in the physically interesting regime where the black hole mass is much smaller than the radius of curvature of the spacetime (which is a relevant regime because the cosmological constant is small). The SdS black hole shows QNM behaviour similar to the Schwarzschild black hole at intermediate times, and soon changes to a power law decay. At late times it again shows QNM behaviour (exponential decay) where the QNMs are now proportional to the surface gravity of the cosmological horizon. For the Schwarzschild black hole, analytic approximations have been used to give a general (approximate) formula for the QNMs as a function of the black hole parameters and the angular mode [9]. It is of interest to compute, in the same spirit, the QNMs for the SdS black hole in an analytical approximation scheme in the physically relevant regime. The advantage is that there exist known numerical studies which reveal characteristic features in the time decay of a field in the SdS black hole. The analytical approximation could be refined to reproduce these features, and at the same time would give a general expression for the QNMs as a function of the spacetime parameters and the angular mode. The approximate model, being simpler to study may also be possible to analyse

* e-mail: suneeta@theorie.physik.uni-muenchen.de 
completely. It would thus give insight into the qualitative features of the complete time behaviour of the field in SdS spacetime. Further, the near-extremal limit and its effect on the QNMs and time behaviour of fields could be explored in this model.

In the recent past, the QNMs of Anti-de Sitter (AdS) black holes have been studied extensively. A detailed numerical study of QNM decay of scalar fields in AdS black hole backgrounds in various dimensions was performed in [10]. Exact computations of QNMs for the BTZ black hole in $(2+1)$ dimensions were demonstrated first in [11] and subsequently higher order modes (as also numerical results for gravitational perturbations of SAdS black holes) are shown in 12]. More general numerical computations of QNMs for higher dimensional AdS black holes are found in 13 . Decay of scalar fields coupled to curvature in topological AdS black hole backgrounds is studied numerically in 14, 15] and analytically in [16]. An interpretation of the QNMs for an AdS black hole in the dual CFT using the AdS/CFT correspondence is provided in [17] (see also [18]). An interesting question is whether the QNMs of the SdS black hole have an interpretation in a dual CFT using the recently proposed dS/CFT correspondence 19] (for a study of pure de Sitter QNMs in this context, see [20]). The study of the time behaviour of perturbations in an analytical model for the SdS spacetime could be used to study such a possible interpretation.

In this paper, we propose an analytical model to study the time behaviour of perturbations in the SdS black hole. We compute the QNMs of this model in the regime when the black hole mass is small compared to the radius of curvature of the spacetime. The SdS spacetime is bounded by the black hole and cosmological horizon. Our analytical approximation consists of approximating the SdS potential by a potential that reproduces its asymptotic behaviour at both boundaries. In section 2, we enumerate the QNM boundary conditions for the SdS black hole, and describe our approximation potential along with the motivations for our choice. The chosen potential has a discontinuity in its second derivative at the maximum. In section 3, we describe the exact solutions to this potential and list the correct matching coefficients at the maximum of the potential. The actual computation of the QNMs is done in the regime of interest in the next section. Two sets of QNMs, one proportional to the black hole horizon surface gravity and the other proportional to the surface gravity of the cosmological horizon are found - the first set describing the time behaviour of the field at certain intermediate times, and the latter describing late-time behaviour. A qualitative picture for the time scales where each of these two sets of QNMs comes into play is given - and it is discussed why this behaviour is expected only in the regime where the black hole mass is small compared to radius of curvature. The real part of the QNMs depends on the maximum of the potential. Our approximation consists of identifying the value of the maximum of the model potential with that of the SdS potential. We compute the maximum value of the SdS potential in section 5. This unfortunately is difficult to do for any general angular mode. We therefore consider the small angular mode and large angular mode cases separately while computing the SdS maximum. In our last section, we present a detailed comparison of the results of this model with numerical studies. There is good qualitative agreement in the most striking feature of the numerical studies, the presence of the two sets of QNMs relevant at two different time scales, and which are proportional to the black hole and cosmological horizon surface gravities respectively. We also show in this section, non-completeness of QNMs in our model - that is also expected from the observation of power law behaviour at intermediate times for the SdS black hole. We discuss how the model could be refined or modified to reproduce the more quantitative features of the numerical results like the dependence of the QNMs on the angular mode. We also discuss the exciting questions which can be addressed for this model, and would shed light on our understanding of perturbations of the SdS spacetime.

\section{ANALYTICAL APPROXIMATION OF THE SDS POTENTIAL}

The metric for the Schwarzschild-de Sitter spacetime in four dimensions for cosmological constant $\Lambda=3 / l^{2}$ is given by

$$
d s^{2}=-(N)^{2} d t^{2}+(N)^{-2} d r^{2}+r^{2}(d \Omega)^{2}
$$

with

$$
N=\sqrt{\left(1-\frac{2 M}{r}-\frac{r^{2}}{l^{2}}\right)} .
$$

There are now two horizons given by the zeroes of $N^{2}$. These are the two real positive roots of $N^{2}$,

$$
r_{b}=\frac{2}{\sqrt{\Lambda}} \sin \left[\frac{1}{3} \sin ^{-1}(3 M \sqrt{\Lambda})\right] \text { and }
$$




$$
r_{c}=\frac{2}{\sqrt{\Lambda}} \sin \left[\frac{1}{3} \sin ^{-1}(3 M \sqrt{\Lambda})+\frac{2 \pi}{3}\right]
$$

The third root of $N^{2}$ is negative, and given by $r_{0}=-\left(r_{b}+r_{c}\right) . r_{b}$ is the black hole horizon and $r_{c}$ the cosmological horizon. As in the asymptotically flat case, the spacetime has a curvature singularity at $r=0$.

We consider a massless scalar field $\Phi$ in this background. Then, the Klein-Gordon equation for the field is

$$
\partial_{\mu}\left(\sqrt{-g} g^{\mu \nu} \partial_{\nu}\right) \Phi=0
$$

Using an ansatz for the field

$$
\Phi=\frac{1}{r} \chi(r) e^{i \omega t} Y_{L M}(\theta, \phi),
$$

and by going to the tortoise coordinate $x$ where $d x=d r / N^{2}$, the Klein-Gordon equation is

$$
-\frac{d^{2} \chi}{d x^{2}}+V_{L}(r) \chi=\omega^{2} \chi
$$

where

$$
V_{L}(r)=\left(1-\frac{2 M}{r}-\frac{r^{2}}{l^{2}}\right)\left(\frac{2 M}{r^{3}}-\frac{2}{l^{2}}+\frac{L(L+1)}{r^{2}}\right)
$$

When $r=r_{b}, x=-\infty$. When $r=r_{c}, x=\infty$. We are interested in studying scalar field perturbations in the region bounded by these two horizons. The potential $V_{L}(r)$ goes exponentially to zero as a function of the $x$ coordinate as one approaches the two horizons. However, the rate of approach is not the same - it depends on the surface gravities associated with the horizons. The surface gravity at a horizon $r_{h}$ is defined as $\alpha_{h}=\frac{1}{2}|d f / d r|_{r_{h}}$. Thus the surface gravities associated with the black hole and cosmological horizons, $\alpha_{b}$ and $\alpha_{c}$ respectively are

$$
\begin{aligned}
\alpha_{b} & =\frac{\left(r_{c}-r_{b}\right)\left(r_{b}-r_{0}\right)}{2 l^{2} r_{b}} \text { and } \\
\alpha_{c} & =\frac{\left(r_{c}-r_{b}\right)\left(r_{c}-r_{0}\right)}{2 l^{2} r_{c}} .
\end{aligned}
$$

The potential $V_{L}(r)$ goes to zero as $e^{2 \alpha_{b} x}$ as $x \rightarrow-\infty$, i.e as the black hole horizon is approached. When $x \rightarrow \infty$, i.e as the cosmological horizon is approached, $V_{L}(r)$ goes to zero as $e^{-2 \alpha_{c} x}$. In studying the time evolution of the perturbation, it is important to first compute the QNMs for the problem. QNMs are given in this case by solutions to (6) with specific boundary conditions - ingoing at the black hole horizon, and outgoing at the cosmological horizon. These are the physically motivated boundary conditions for the problem as a timelike observer in this spacetime can send messages, but not receive them through the (future) cosmological horizon. The boundary condition at the black hole horizon is the usual one for computing QNMs of black holes and represents the classical absorption by the black hole. The QNM boundary conditions for the field are thus:

$$
\begin{aligned}
& \chi \propto \exp (i \omega x) ; \quad x \rightarrow-\infty \text { and } \\
& \chi \propto \exp (-i \omega x) ; \quad x \rightarrow+\infty .
\end{aligned}
$$

$\omega$ is now complex and $\omega=\omega_{0}+i \Gamma$, where $\Gamma>0$.

Thus, the QNMs can be obtained by solving (6) with the above boundary conditions. However, exact solutions to this equation are not known. In fact, such exact solutions are not available even for the Schwarzschild black hole in an asymptotically flat spacetime. Instead, what is done in the asymptotically flat case is to either solve the equation numerically and compute the QNMs, or to analytically approximate the Schwarzschild potential by an exactly solvable potential. This potential is described by parameters which can be used to fit the potential to the Schwarzschild potential [9]. We follow the second approach of an analytic approximation in computing the QNMs of the SdS black hole. 
In our search for a potential that would be a good analytic approximation to the SdS potential, we set out to reproduce the behaviour of the SdS potential as $x \rightarrow \pm \infty$. The approximate potential $V$ that we have chosen has the following features :

1. Its second derivative is discontinuous at $r=r_{m}$, where $r_{m}$ is the value of $r$ for which the actual SdS potential has a maximum, i.e a solution of $d V_{L} / d r=0$.

2. In the region $r_{b}<r \leq r_{m}$, the potential is

$$
V_{1}=\frac{V_{m}}{\left[\cosh \alpha_{b}\left(x-x_{m}\right)\right]^{2}} .
$$

$x_{m}$ is the value of the $x$ coordinate when $r=r_{m} . \alpha_{b}$ is given by (9). $V_{1}$ is referred to as the Poschl-Teller potential in literature, and was first used in [9] to approximate the Schwarzschild potential. $V_{m}$ is taken to be the maximum of the SdS potential.

3. In the region $r_{m} \leq r<r_{c}$, the potential is taken to be again the Poschl-Teller potential, but with a different choice of parameter. It is

$$
V_{2}=\frac{V_{m}}{\left[\cosh \alpha_{c}\left(x-x_{m}\right)\right]^{2}} .
$$

$\alpha_{c}$ is given by (9).

As can be checked, this potential reproduces the asymptotic form of the SdS potential at the two boundaries. Furthermore, this potential is exactly solvable. Therefore what remains is a computation of $V_{m}$, the value of the SdS potential at its maximum. Computing the maximum of the SdS potential is difficult as the potential is complicated. We are forced to work in the regime $M \ll l$. The Poschl-Teller has been used to approximate the SdS potential recently [5]. However, in this case, one Poschl-Teller potential is used throughout - and this is a good approximation only for the nearly extreme SdS black hole, where the two horizons are very close (i.e, $M$ and $l$ are of the same order) 6]. In the other regime $M \ll l$, one Poschl-Teller potential provides a poor approximation to the SdS potential as the asymptotic forms of the SdS potential at both the boundaries cannot be reproduced by it. This problem is solved by our choice for the potential, $V$. It would be exciting if the near-extremal limit in our model led to the QNMs found in [6]. We must now check what features, qualitative or quantitative, we reproduce of numerical studies of dynamics of a scalar field in the SdS spacetime done in [7, 8] by a study of this potential.

We now proceed to compute the QNMs of the approximate potential $V$ which is given by $V_{1}$ for $r \leq r_{m}$ and $V_{2}$ for $r \geq r_{m}$. We are interested in the solutions to the equation :

$$
-\frac{d^{2} \chi}{d x^{2}}+V \chi=\omega^{2} \chi .
$$

For both $V_{1}$ and $V_{2}$, exact solutions can be found. We first examine the exact solutions to (13) with both $V=V_{1}$ and $V=V_{2}$. Then, the solutions have to be matched at $r=r_{m}$. Finally, we must pick the solutions that obey the QNM boundary conditions at both the boundaries.

\section{EXACT SOLUTIONS FOR THE POTENTIAL}

Let us consider the solutions to (13) with $V=V_{1}$. The solutions to this (Poschl-Teller) potential are known. They can be written in terms of the hypergeometric functions after a change of variables to $\xi$ where $\xi /(1-\xi)=e^{2 \alpha_{b}\left(x-x_{m}\right)}$. Then, two linearly independent solutions are :

$$
\begin{aligned}
& \chi_{1}=[\xi(1-\xi)]^{\frac{i \omega}{2 \alpha_{b}}} F(a, b, c ; \xi) \text { and } \\
& \chi_{2}=[\xi(1-\xi)]^{\frac{i \omega}{2 \alpha_{b}}}(\xi)^{1-c} F(a-c+1, b-c+1,2-c ; \xi) .
\end{aligned}
$$


We have

$$
a=\frac{1}{2}+\sqrt{\frac{1}{4}-\frac{V_{m}}{\alpha_{b}^{2}}}+\frac{i \omega}{\alpha_{b}}, b=\frac{1}{2}-\sqrt{\frac{1}{4}-\frac{V_{m}}{\alpha_{b}^{2}}}+\frac{i \omega}{\alpha_{b}}, c=1+\frac{i \omega}{\alpha_{b}} .
$$

The black hole horizon is at $\xi=0$. At the maximum of the potential, when $x=x_{m}, \xi=1 / 2$.

Solutions to (13) with $V=V_{2}$ are similar to the previous case, but with $\alpha_{b}$ replaced by $\alpha_{c}$.

Define $\xi_{1}$ where $\xi_{1} / 1-\xi_{1}=e^{2 \alpha_{c}\left(x-x_{m}\right)}$.

$$
\begin{aligned}
& \tilde{\chi}_{1}=\left[\xi_{1}\left(1-\xi_{1}\right)\right]^{\frac{i \omega}{\alpha_{c}}} F\left(a_{1}, b_{1}, c_{1} ; \xi_{1}\right) \text { and } \\
& \tilde{\chi}_{2}=\left[\xi_{1}\left(1-\xi_{1}\right)\right]^{\frac{i \omega}{2 \alpha_{c}}}\left(\xi_{1}\right)^{1-c_{1}} F\left(a_{1}-c_{1}+1, b_{1}-c_{1}+1,2-c_{1} ; \xi_{1}\right) .
\end{aligned}
$$

Here

$$
a_{1}=\frac{1}{2}+\sqrt{\frac{1}{4}-\frac{V_{m}}{\alpha_{c}^{2}}}+\frac{i \omega}{\alpha_{c}}, b_{1}=\frac{1}{2}-\sqrt{\frac{1}{4}-\frac{V_{m}}{\alpha_{c}^{2}}}+\frac{i \omega}{\alpha_{c}}, c_{1}=1+\frac{i \omega}{\alpha_{c}} .
$$

The next step is to match an arbitrary superposition of the two solutions (15) and (15) with an arbitrary superposition of (18) and (18) - at $x=x_{m}$. This involves matching both the wave functions and their first derivatives across $x=x_{m}$. Let us use the following notation: $\chi_{1}\left(x_{m}\right)=X, \quad \chi_{2}\left(x_{m}\right)=Y$. Also, $\quad \frac{d \chi_{1}}{d x}\left(x_{m}\right)=X^{\prime}, \quad \frac{d \chi_{2}}{d x}\left(x_{m}\right)=Y^{\prime}$. $\tilde{\chi}_{1}\left(x_{m}\right)=\tilde{X}, \quad \tilde{\chi}_{2}\left(x_{m}\right)=\tilde{Y}$, and $\frac{d \tilde{\chi}_{1}}{d x}\left(x_{m}\right)=\tilde{X}^{\prime}, \quad \frac{d \tilde{\chi}_{2}}{d x}\left(x_{m}\right)=\tilde{Y}^{\prime}$.

Then matching the wave functions and their derivatives across $x=x_{m}$ yields

$$
A X+B Y=\tilde{A} \tilde{X}+\tilde{B} \tilde{Y} ; A X^{\prime}+B Y^{\prime}=\tilde{A} \tilde{X}^{\prime}+\tilde{B} \tilde{Y}^{\prime} .
$$

Here, $A, B, \tilde{A}$ and $\tilde{B}$ are arbitrary coefficients of superposition of the two linearly independent solutions, which are then $\chi=A \chi_{1}+B \chi_{2}$ for $r<r_{m}$ and $\chi=\tilde{A} \tilde{\chi}_{1}+\tilde{B} \tilde{\chi}_{2}$ for $r>r_{m}$.

$A$ and $B$ are related to $\tilde{A}$ and $\tilde{B}$ as :

$$
\begin{aligned}
& \tilde{A}=A \frac{\left(X \tilde{Y}^{\prime}-X^{\prime} \tilde{Y}\right)}{\left(\tilde{X} \tilde{Y}^{\prime}-\tilde{X}^{\prime} \tilde{Y}\right)}+B \frac{\left(Y \tilde{Y}^{\prime}-Y^{\prime} \tilde{Y}\right)}{\left(\tilde{X} \tilde{Y}^{\prime}-\tilde{X}^{\prime} \tilde{Y}\right)} \\
& \tilde{B}=A \frac{\left(X \tilde{X}^{\prime}-X^{\prime} \tilde{X}\right)}{\left(\tilde{Y} \tilde{X}^{\prime}-\tilde{Y}^{\prime} \tilde{X}\right)}+B \frac{\left(Y \tilde{X}^{\prime}-Y^{\prime} \tilde{X}\right)}{\left(\tilde{Y} \tilde{X}^{\prime}-\tilde{Y}^{\prime} \tilde{X}\right)} .
\end{aligned}
$$

We recognise that at $x=x_{m}$, the value of $\xi$ that appears in (15) and (15) is $1 / 2$. Using this and properties of the hypergeometric functions, we can write :

$$
\begin{aligned}
X & =\sqrt{\pi} \exp \left(-\frac{i \omega \ln 2}{\alpha_{b}}\right) \frac{\Gamma\left(\frac{1}{2}+\frac{a}{2}+\frac{b}{2}\right)}{\Gamma\left(\frac{1}{2}+\frac{a}{2}\right) \Gamma\left(\frac{1}{2}+\frac{b}{2}\right)}, \\
X^{\prime} & =\frac{\sqrt{\pi} \alpha_{b}}{2} \exp \left(-\frac{i \omega \ln 2}{\alpha_{b}}\right) \frac{a b}{c} \frac{\Gamma\left(2+\frac{i \omega}{\alpha_{b}}\right)}{\Gamma\left(1+\frac{a}{2}\right) \Gamma\left(1+\frac{b}{2}\right)}, \\
Y & =\sqrt{\pi} \exp \left(\frac{i \omega \ln 2}{\alpha_{b}}\right) \frac{\Gamma\left(1-\frac{i \omega}{\alpha_{b}}\right)}{\Gamma\left(1-\frac{a}{2}\right) \Gamma\left(1-\frac{b}{2}\right)}, \\
Y^{\prime} & =-i \omega \sqrt{\pi} \exp \left(\frac{i \omega \ln 2}{\alpha_{b}}\right) \frac{\Gamma\left(1-\frac{i \omega}{\alpha_{b}}\right)}{\Gamma\left(1-\frac{a}{2}\right) \Gamma\left(1-\frac{b}{2}\right)} \\
& +\frac{\alpha_{b}}{2} \frac{(a-c+1)(b-c+1)}{2-c} F\left(a-c+2, b-c+2,3-c ; \frac{1}{2}\right) .
\end{aligned}
$$


$\tilde{X}, \tilde{Y}, \tilde{X}^{\prime}$ and $\tilde{Y}^{\prime}$ are given by the same expressions as above, except that we replace $\alpha_{b}$ with $\alpha_{c}$. Consequently $a, b$ and $c$ are also replaced by their counterparts $a_{1}, b_{1}$ and $c_{1}$. We therefore do not display those expressions here.

\section{COMPUTATION OF QUASINORMAL MODES}

We search for solutions to the problem satisfying quasinormal mode boundary conditions at the black hole and cosmological horizons given by (10). The solution obeying the QNM boundary conditions at the black hole horizon $\chi_{q n m}$ for $x>x_{m}$ is given by $\tilde{A} \tilde{\chi}_{1}+\tilde{B} \tilde{\chi}_{2}$. $\tilde{A}$ and $\tilde{B}$ for $\chi_{q n m}$ are given by (21) and (22) with $B=0$. Near the cosmological horizon, i.e as $x \rightarrow \infty$, this solution is

$$
\chi_{q n m} \sim \tilde{A} P_{1} \exp (-i \omega x)+\tilde{A} Q_{1} \exp (i \omega x)+\tilde{B} P_{2} \exp (-i \omega x)+\tilde{B} Q_{2} \exp (i \omega x),
$$

where

$$
\begin{aligned}
P_{1} & =\frac{\Gamma\left(c_{1}\right) \Gamma\left(c_{1}-a_{1}-b_{1}\right)}{\Gamma\left(c_{1}-a_{1}\right) \Gamma\left(c_{1}-b_{1}\right)}, \\
Q_{1} & =\frac{\Gamma\left(c_{1}\right) \Gamma\left(a_{1}+b_{1}-c_{1}\right)}{\Gamma\left(a_{1}\right) \Gamma\left(b_{1}\right)}, \\
P_{2} & =\frac{\Gamma\left(2-c_{1}\right) \Gamma\left(c_{1}-a_{1}-b_{1}\right)}{\Gamma\left(1-a_{1}\right) \Gamma\left(1-b_{1}\right)}, \\
Q_{2} & =\frac{\Gamma\left(2-c_{1}\right) \Gamma\left(a_{1}+b_{1}-c_{1}\right)}{\Gamma\left(a_{1}-c_{1}+1\right) \Gamma\left(b_{1}-c_{1}+1\right)} .
\end{aligned}
$$

For (27) to obey QNM boundary conditions at the cosmological horizon given by (10), we must have

$$
\tilde{A} Q_{1}+\tilde{B} Q_{2}=0
$$

Substituting for $\tilde{A}$ and $\tilde{B}$ from (21) and (22) with $B=0$, the above equation is

$$
\left(X \tilde{Y}^{\prime}-X^{\prime} \tilde{Y}\right) \frac{\Gamma\left(c_{1}\right)}{\Gamma\left(a_{1}\right) \Gamma\left(b_{1}\right)}-\left(X \tilde{X}^{\prime}-X^{\prime} \tilde{X}\right) \frac{\Gamma\left(2-c_{1}\right)}{\Gamma\left(a_{1}-c_{1}+1\right) \Gamma\left(b_{1}-c_{1}+1\right)}=0 .
$$

The special frequencies $\omega$ that solve the above equation are the quasinormal mode frequencies for this problem. However, as can be seen from the expressions for $X$ and $X^{\prime}$ from (23) and (24) - the zeroes of $X$ and $X^{\prime}$ do not occur for the same set of frequencies. The zeroes of the above equation cannot therefore be computed simply. However, here we reflect on what we must expect. From the numerical work in [7, 8], we note the presence of two different patterns of exponential decay of the field with time. For intermediate times, the field decays exponentially with the exponent proportional to the black hole surface gravity. This is a typical QNM time behaviour where the QNM frequency is proportional to the black hole surface gravity. This temporal behaviour is similar to that of a field in a Schwarzschild black hole background. This exponential decay is followed by a power law decay of the field - but for late times, there is a switch back to exponential decay. Now the exponent is proportional to the surface gravity of the cosmological horizon. This exponential decay is again a QNM time behaviour, but the QNMs are now proportional to the surface gravity of the cosmological horizon. This numerical result was seen for a choice of well separated length scales $M$ and $l$.

We expect a similar behaviour (i.e two sets of QNMs) for our approximate potential $V$. At very early times, there is a direct propagation of the perturbation without scattering by the potential. At later times, the perturbation gets multiply scattered by the potential. It therefore starts reflecting the properties of the potential. The time behaviour of the field here is dominated by the QNMs. In our case, the second derivative of the potential has a discontinuity at $r=r_{m}$. The QNMs which we have obtained (and which are supposed to describe the time evolution after the very early times) also reflect this. When the perturbation gets scattered for $r_{b}<r \leq r_{m}$ by the potential (which is now $V_{1}$ ), its time evolution will be governed by QNMs related to $V_{1}$ which should be proportional to $\alpha_{b}$ (as is typical for QNMs of a Poschl Teller potential). A rough estimate for the times when this occurs is the light crossing time 
to traverse a distance of the order of $r_{m}$, i.e $r_{m} / c$. When the perturbation travels a distance much greater than $r_{m}$, it starts getting scattered by the potential $V_{2}$, and its time evolution is governed by QNMs related to $V_{2}$, which we expect to be proportional to $\alpha_{c}$. This roughly occurs at times of the order of the light crossing time to traverse a distance $l$, i.e $l / c$. At such times and later, the decay of the perturbation is governed solely by the QNMs related to $V_{2}$. However, the above statements are possible only when there is a clear separation of these two interesting time scales. As we see in the next section, if we have a clear separation of the length scales $M$ and $l$, this implies that $r_{m} \sim O(M)$. Thus a clear separation of the two interesting time scales $\frac{r_{m}}{c}$ and $\frac{l}{c}$ is then related to a clear separation of $\alpha_{c} \sim 1 / l$ and $\alpha_{b} \sim 1 / M$. We note that when such a clear separation of the scales does not exist (as for e.g in the nearly extreme SdS black hole studied in [5, 6]) then we do not expect two different sets of QNMs.

Let us return to (33). Since we have not yet specialised to the case where the length scales are separated, we do expect the general solutions of this equation for $\omega$ to be complicated. However, if we specialise to the case where $M \ll l$, and work in an approximation where we can neglect $O(M / l)$ terms and higher, we must see solutions $\omega$ that split into two sets as argued above. We therefore proceed to study the various terms in (33) and drop terms that can be neglected in this approximation. For $M \ll l, \alpha_{b} \sim 1 / M$ and $\alpha_{c} \sim 1 / l$. Solutions $\omega$ for the case of the two length scales being well-separated are of the form $\omega \sim O(1 / M)$ or $\omega \sim O(1 / l)$.

We now look for solutions of the form $\omega \sim O(1 / M)$. Then, in (33), the first term has a factor $\left(X \tilde{Y}^{\prime}-X^{\prime} \tilde{Y}\right)$. From the detailed expression for this factor, it is clear that this factor is proportional to $\exp (i \omega l \ln 2)$. Now, $\omega=\omega_{0}+i \Gamma$ where $\Gamma>0$. Furthermore, $\Gamma \sim O(1 / M)$. Therefore, this factor $\left(X \tilde{Y}^{\prime}-X^{\prime} \tilde{Y}\right)$ is proportional to $\exp (-l / M)$. On the other hand, the second term in (33) has a factor $\left(X \tilde{X}^{\prime}-X^{\prime} \tilde{X}\right)$ which is proportional to $\exp (l / M)$. Since $M \ll l$, the first term can be neglected compared to the second term (Here, we have also used the asymptotic expansion of the hypergeometric function for large parameter). Therefore, in this approximation we must solve the equation

$$
X \tilde{X}^{\prime}-X^{\prime} \tilde{X}=0 \text {. }
$$

Here again, we look at the detailed expressions for $X^{\prime}$ in (24) and $\tilde{X}^{\prime}$. Using these detailed expressions and multiplying (34) by $M$, we see that the first term in (34) $X \tilde{X}^{\prime}$ is $O(M / l)$ compared to the second term $X^{\prime} \tilde{X}$. Neglecting the first term, we see that we must now look for zeroes of the second term which lead to frequencies $\omega \sim O(1 / M)$. These are given by the zeroes of $X^{\prime}$, which occur (as can be seen on inspecting (24) when $1+a / 2=-n$ and $n \geq 0$ is an integer. Thus, the frequencies $\omega$ are :

$$
\omega=\alpha_{b}\left[\sqrt{\frac{V_{m}}{\alpha_{b}^{2}}-\frac{1}{4}}+i\left(2 n+\frac{5}{2}\right)\right] .
$$

We note, that above, we do not consider zeroes of $\tilde{X}$ as they lead to frequencies not of $O(1 / M)$ as assumed while arriving at (34). We have nevertheless shown that in the $M \ll l$ approximation, there indeed exist solutions $\omega \sim$ $O(1 / M)$ to (33).

We now consider the other possibility, i.e $\omega \sim O(1 / l)$. Now we can no longer neglect the first term of (33) compared to the second term, as we did before. However, we multiply the whole equation by $M$, and observe that part of the second term can still be neglected as in the previous case of $\omega \sim O(1 / M)$. More precisely, the second term has a factor $\left(X \tilde{X}^{\prime}-X^{\prime} \tilde{X}\right)$ where the first term is $O(M / l)$ compared to the second, and can be dropped. We now concentrate on the approximate equation

$$
\left(X \tilde{Y}^{\prime}-X^{\prime} \tilde{Y}\right) \frac{\Gamma\left(c_{1}\right)}{\Gamma\left(a_{1}\right) \Gamma\left(b_{1}\right)}+X^{\prime} \tilde{X} \frac{\Gamma\left(2-c_{1}\right)}{\Gamma\left(a_{1}-c_{1}+1\right) \Gamma\left(b_{1}-c_{1}+1\right)}=0 .
$$

There are more $O(M / l)$ terms left in the above equation. However, we observe that the first term has a factor $1 / \Gamma\left(a_{1}\right)$. Thus zeroes of the above equation where $\omega \sim O(1 / l)$ could occur at poles of $\Gamma\left(a_{1}\right)$ which are also zeroes of $\tilde{X}$. We check for such solutions. The poles of $\Gamma\left(a_{1}\right)$ occur when $\Gamma\left(a_{1}\right)=-n^{\prime}$ and $n^{\prime} \geq 0$ is an integer. The corresponding frequencies are

$$
\omega=\alpha_{c}\left[\sqrt{\frac{V_{m}}{\alpha_{c}^{2}}-\frac{1}{4}}+i\left(n^{\prime}+\frac{1}{2}\right)\right] .
$$

The zeroes of $\tilde{X}$ occur for $a_{1}+1=-2 n$ where $n \geq 0$ is an integer. The corresponding frequencies are

$$
\omega=\alpha_{c}\left[\sqrt{\frac{V_{m}}{\alpha_{c}^{2}}-\frac{1}{4}}+i\left(2 n+\frac{3}{2}\right)\right]
$$


From (37) and (38), we see that the poles of $\Gamma\left(a_{1}\right)$ which are also zeroes of $\tilde{X}$ occur when, $n^{\prime}$ is an odd integer. These frequencies $\omega \sim O(1 / l)$ are then the solutions of (36) and in the $M \ll l$ approximation, the solutions of (33).

\section{MAXIMUM OF THE SDS POTENTIAL}

In the previous section, we argued for the presence of two sets of QNMs, one proportional to $\alpha_{b}$ and the other to $\alpha_{c}$, in the approximation $M \ll l$. We also computed these frequencies which are given by (35) and (38). But it remains to evaluate $V_{m}$, the maximum of the $\operatorname{SdS}$ potential $V_{L}(r)$. The maximum is a solution of the equation $d V_{L} / d r=0$, which is a sextic equation in $r$. For high values of the angular mode number $L$, the potential is approximately

$$
V_{L}(r) \sim\left(1-\frac{2 M}{r}-\frac{r^{2}}{l^{2}}\right) \frac{L(L+1)}{r^{2}} .
$$

Then the maximum occurs at $r_{m}=3 M$. Thus, for large $\mathrm{L}$, we can take $V_{m}=V_{L}(3 M)$.

$$
V_{L}(3 M)=\left(\frac{1}{3}-\frac{9 M^{2}}{l^{2}}\right)\left(\frac{2}{27 M^{2}}-\frac{2}{l^{2}}+\frac{L(L+1)}{9 M^{2}}\right) \text {. }
$$

Let us now look at small values of $L$. We first consider the case $L=0$. For $L=0$, numerically, with $M=1$ and $\Lambda=10^{-4}$, we can estimate the maximum, which is $r_{m}=2.67$. For $M=2$ and the same value of $\Lambda, r_{m}=5.33$. This suggests that $r_{m}=2.67 M$. Analytically, the equation to be solved is $d V_{0} / d r=0$, and the equation is

$$
2 \frac{r^{6}}{l^{6}}-\frac{M r^{3}}{l^{4}}-\frac{3 M r}{l^{2}}+\frac{8 M^{2}}{l^{2}}=0 .
$$

Now, let us assume that the solution $r_{m} \sim O(M)$ as suggested by our numerical results. Then, in the equation above, the first and second terms are $O\left((M / l)^{2}\right)$ or higher powers of $(M / l)$ compared to the last two terms. Therefore, dropping these terms we see that $r_{m}=\frac{8}{3} M$. This agrees with our numerical results. For the QNMs with $L=0$, we can therefore set $V_{m}=V_{0}\left(\frac{8}{3} M\right)$.

$$
V_{0}\left(\frac{8}{3} M\right)=\left(\frac{1}{4}-\frac{64 M^{2}}{9 l^{2}}\right)\left(\frac{27}{256 M^{2}}-\frac{2}{l^{2}}\right)
$$

For $L=1$, we can again solve the equation $d V_{1} / d r=0$, assuming as before that the solution $r_{m} \sim O(M)$ and in the approximation $M \ll l$. We find that $r_{m}=\frac{1}{4}(3+\sqrt{73}) M$, i.e $r_{m} \sim 2.886 M$. We expect $r_{m}=3 M$ to be a good approximation to the maximum for higher values of $L$.

In all the above cases, we can explicitly check that $V_{m} / \alpha_{b}^{2}$ and $V_{m} / \alpha_{c}^{2}$ are greater than $1 / 4$. Thus, it is the real part of the QNM frequencies that depends on $V_{m}$. Substituting for the value of $V_{m}$ from (40) in the expressions for the QNMs (35) and (38) - we get the explicit expression for the QNM frequencies for $L>0$. For $L=0$, using (42) for $V_{m}$, we obtain more precise values for the QNM frequencies.

\section{DISCUSSION}

In the previous sections, we have done an analysis of a perturbation in the potential $V$ and given a sketch of its behaviour in time. We have found the presence of two sets of QNMs which are relevant at two different time scales (as discussed in Section IV):

$$
\begin{aligned}
& \omega=\alpha_{b}\left[\sqrt{\frac{V_{m}}{\alpha_{b}^{2}}-\frac{1}{4}}+i\left(2 n+\frac{5}{2}\right)\right] \text { and } \\
& \omega=\alpha_{c}\left[\sqrt{\frac{V_{m}}{\alpha_{c}^{2}}-\frac{1}{4}}+i\left(2 n+\frac{3}{2}\right)\right] .
\end{aligned}
$$


$V_{m}$ is determined as discussed in the previous section. We have also given a qualitative picture for the times at which each of these sets of QNMs would be relevant for the temporal behaviour of the scalar field.

We now address the question of how well this reflects the time behaviour of a perturbation in the SdS potential. Numerical studies have been done for scalar fields in the SdS spacetime 7, 8]. We indeed reproduce the most striking feature of the studies, the presence of two sets of modes which are proportional to the surface gravities of the black hole and cosmological horizons relevant at intermediate and late times respectively.

Now let us make more quantitative comparison with the numerical results. We begin with the intermediate times at which the first set of modes (35) are relevant. In the numerical work [8], a scalar field wave function with a Gaussian profile is chosen. Its subsequent time evolution in the SdS spacetime is found by numerically integrating the Klein-Gordon equation. A choice of $r_{b}=1$ and $r_{c}=2000$ (such that we are in the regime $M \ll l$ ) is taken. A graph of the field behaviour in an SdS spacetime versus similar behaviour in a Schwarzschild spacetime is plotted for $L=0$ and $L=1$. It is seen that for early times, the two graphs follow each other closely and display QNM oscillations they start to differ from each other only for times much greater than $\frac{r_{b}}{c}$.

Let us compare our first set of modes (35) with the Schwarzschild QNMs which seem to describe the behaviour in SdS well at these times. To do so, we can use an analytical approximation for the Schwarzschild QNMs by Ferrari and Mashhoon [9] which approximates the QNMs well when their imaginary parts are not too large. Then in this approximation, the real $\left(\omega_{R}^{S}\right)$ and imaginary $\left(\omega_{I m}^{S}\right)$ parts of the Schwarzschild QNMs are $\omega_{R}^{S}=\alpha\left(\sqrt{\frac{U_{0}}{\alpha^{2}}-\frac{1}{4}}\right)$ and $\omega_{I m}^{S}=\alpha\left(n+\frac{1}{2}\right)$. Here, $\alpha$ and $U_{0}$ are given by the height and curvature of the Schwarzschild potential at its maximum and $n$ is an integer. $\alpha^{-1}=3 \sqrt{3} M$. Comparing with the modes (35), we see that for $M \ll l, U_{0} \sim V_{m}$. Also $\alpha_{b}^{-1} \sim 4 M$ and therefore $\alpha_{b} \sim(1.3) \alpha$. Thus we see that the real parts of the Schwarzschild QNMs and (35) have approximately the same $M$ dependence. The angular mode $L$ dependence is also the same in both real parts. However, coming to the imaginary parts, we see that our set of QNMs reproduces only those Schwarzschild QNMs starting from $n=2$ and subsequently reproduces only alternate $n$ modes, the next one being $n=4$. This probably has to do with our matching conditions at $r_{m}$.

We proceed to compare the time behaviour of the field in this model with numerical results for later times. In the next sub-section, we address intermediate time power law decay. The following sub-section compares the QNM behaviour at late times, i.e given by (38) in our model with numerical results - particularly with reference to angular mode dependence.

\section{A. Incompleteness of QNMs}

An important point in the numerical results [7, 8] is the observation of a power law behaviour in time for the field at certain intermediate times, rather than QNM behaviour. It is seen that the time decay of the field first displays Schwarzschild QNM behaviour, then Schwarzschild power law decay. At later times, the power law decay becomes faster (to be followed eventually by exponential decay).

We examine whether there is a possibility of such interesting behaviour in our model. A detailed analysis has been done in 21] on the conditions (and types of potentials) for which both QNM and power law behaviour in time may be present at different times. Also, the more general question of the potentials for which the QNMs form a complete set has been addressed. The results of the analysis are best expressed in terms of the Fourier transform of the Green's function for the system. This can be compactly written in terms of two auxiliary functions $f(\omega, x)$ and $g(\omega, x)$ which are solutions to the homogenous time-independent Klein Gordon equation, where $f$ satisfies the QNM boundary condition at one boundary (in our case, the black hole horizon) and $g$ satisfies the QNM boundary condition at the other boundary (in our case, the cosmological horizon). Then the Green's function is

$$
\begin{aligned}
\tilde{G}(x, y ; \omega) & =f(\omega, x) g(\omega, y) / W(\omega) \text { for } 0<x<y, \\
& =f(\omega, y) g(\omega, x) / W(\omega) \text { for } 0<y<x .
\end{aligned}
$$

Here, the Wronskian $W(\omega)=g(\omega, x) f^{\prime}(\omega, x)-f(\omega, x) g^{\prime}(\omega, x)$ is independent of $x$. The QNMs are given by the 
zeroes of the Wronskian, i.e when the functions $f$ and $g$ are linearly dependent. Following [21], the QNMs do not describe the intermediate or late time behaviour completely when the functions $g$ and $f$ are not analytic w.r.t $\omega$. Rather, non-analyticity of these functions could lead to power law behaviour in time. $f$ and $g$ are analytic in $\omega$ if the potential for the system $V$ has 'no tail' at each of the boundaries $x=-\infty$ and $x=+\infty$ in the following sense (condition for boundary at $x=\infty$ ) :

$$
\int_{0}^{\infty} d x x|V(x)|<\infty, \quad \int_{0}^{\infty} d x x e^{\alpha x}|V(x)|<\infty \text { for any } \alpha>0 .
$$

There is a corresponding condition for the boundary at $x=-\infty$. If this condition is violated for some $\alpha>\alpha_{0}>0$, then $f$ and $g$ may not be analytic in $\omega$ for $\operatorname{Im} \omega>\alpha_{0}$. Let us apply these results to our problem. For our problem, the function $f(\omega, x)$ obeying the QNM boundary condition at the black hole horizon is $\chi_{1}$, and for $x \rightarrow \infty$, it is given by the expression for $\chi_{q n m}$ in (27). The function $g(\omega, x)$ obeying the QNM boundary condition at the cosmological horizon is

$$
g(\omega, x)=\frac{1}{\left(Q_{2} P_{1}-Q_{1} P_{2}\right)}\left(Q_{2} \tilde{\chi}_{1}-Q_{1} \tilde{\chi}_{2}\right) .
$$

It can be easily checked that the Wronskian $W(\omega)$ is

$$
W(\omega)=-2 i \omega\left(\tilde{A} Q_{1}+\tilde{B} Q_{2}\right) .
$$

QNMs are frequencies for which the Wronskian is zero, and as expected, are given by solutions to (32) which we discussed extensively in the previous sections.

We are interested in the question of whether $f$ and $g$ are analytic in $\omega$. The potential $V$ for our problem goes to zero as $e^{-2 \alpha_{c} x}$ as $x \rightarrow \infty$ and as $e^{2 \alpha_{b} x}$ as $x \rightarrow-\infty$. Therefore, conditions (46) are violated for $\alpha>\alpha_{b}$ (coming from the condition at the black hole horizon) and for $\alpha>\alpha_{c}$ (coming from the condition at the cosmological horizon). We expect non-analyticity for two sets of $\omega: \operatorname{Im} \omega>\alpha_{b}$ and $\operatorname{Im} \omega>\alpha_{c}$.

Let us now examine $f$ and $g$ to see if this is indeed the case. $f=\chi_{1}$. Using the properties of hypergeometric functions for the specific values of parameters $a, b$ and $c$ for the problem, we can write $f$ in terms of the associated Legendre polynomial as

$$
\chi_{1}=[\xi(1-\xi)]^{\frac{i \omega}{2 \alpha_{b}}} \Gamma(c)\left(\xi-\xi^{2}\right)^{(1-a-b) / 4} P_{(a-b-1) / 2}^{(1-a-b) / 2}(1-2 \xi) .
$$

Therefore $f$ is not analytic for $c=-n$ where $n \geq 0$ is an integer. This occurs for $\omega=i \alpha_{b}(n+1)$. From (47), the poles of $g$ are given by the poles of $Q_{1} / Q_{2}$, and therefore occur when $c_{1}=-n$. The corresponding frequencies $\omega=i \alpha_{c}(n+1)$. Thus non-analyticity of $f$ and $g$ are as expected from the violations of (46). This non-analyticity implies from the results in 21] that QNMs do not completely describe the time behaviour of the scalar field at intermediate times for the potential $V$. Since we have chosen our potential to match the SdS potential asymptotically, we expect the same result for the SdS potential. The numerical results for a field in the SdS potential suggest power law behaviour in time for certain intermediate times. It would be interesting to see if such a power law behaviour could be derived in our model and the parameters the exponent would depend on. In particular, it would be useful if in our model, we could completely obtain the time dependence of the field and the times at which the power law behaviour sets in. This would be a valuable pointer to the time decay of the field in the SdS spacetime.

\section{B. Angular mode dependence of late time behaviour}

We now compare the late time behaviour in our model with numerical results. The late time behaviour in the SdS spacetime was first studied in [7] and later in [8]. The numerical results consider (as for the early times) a scalar field wave function with certain initial conditions whose time evolution is determined by numerically integrating the Klein-Gordon equation. However, as it is required to access late times, it is no longer possible to work with the choice of black hole parameters used for early times, and the authors in [8] use $r_{b}=1$ and $r_{c}=100$ - but now the two horizons are not so well-separated. They then study the leading late time behaviour for the angular modes $L=0,1,2$. For $L=0$, they find that the field settles to a constant value. For $L=1,2$, the field decays exponentially with 
the argument of the exponent consistent with the formula $-\alpha_{c} L$. However, it is not possible for them to verify this proposed formula for the higher angular modes $(L>2)$ as the numerical integration becomes noisy. Thus the late time behaviour (being exponential decay) is a typical QNM behaviour where the QNM is pure imaginary. However, the numerical results display only the leading behaviour, given by the lowest QNM which seems to be $\omega=i \alpha_{c} L$. It must be noted that no numerical results are available on the nature of the higher order modes for a given $L$ (although the problem is addressed by the authors of [8] in an analytical approximation where the central black hole in the SdS spacetime is disregarded).

We consider the QNMs describing late time behaviour in our model given by (38). These QNMs are proportional to $\alpha_{c}$ and have a non-zero real part which depends on $L$. The dependence of the QNMs on the angular mode $L$ is through the maximum of the potential $V_{m}$ and therefore affects only their real part. This is very similar to results obtained for the nearly extreme SdS black hole in [5, 6]. However it is at variance to the numerical results in [8] where at least for the lowest QNM, the real part is zero, and the imaginary part depends on $L$ (although both the model QNMs and the numerical results show a dependence on $\alpha_{c}$ ). Unfortunately, we also have no numerical results available on higher order modes (for a given $L$ ) to compare with the higher order model QNMs.

Our approximate potential $V$ is the simplest model which is solvable in the limit of separation of scales, and reveals the presence of two sets of QNMs coming into play at intermediate and late times as seen in numerical studies. However, it does need to be refined further to also reproduce the $L$ dependence of the second set of modes. However, the refinement has to be such that the model remains tractable at least in the physical regime of interest. This is a severe restriction. This is because much of our success above in reproducing the striking qualitative features has to do with correctly reproducing the asymptotic behaviour of the SdS potential at both horizons. The SdS potential falls off exponentially at both horizons, but with different arguments. This is difficult to reproduce in an exactly solvable potential, and therefore, one must piece together two potentials as we have done at a point, which could be the maximum of the potential. However, in this case, matching conditions may make the actual equation for QNMs complicated. The $L$ dependence seen in numerical studies for the second set of modes and the failure of this model to reproduce them is related to the failure of a Poschl-Teller potential to describe the $L$ dependence of the vacuum de Sitter potential. One obvious modification we could do to our model to reproduce the $L$ dependence correctly is to use the same Poschl-Teller potential for $r_{b}<r<r_{m}$ and the vacuum de Sitter potential for $r_{m}<r<r_{c}$. However, in that case, the matching conditions at $r_{m}$ become very complicated. This makes the equation for QNMs complicated, and it is no longer possible to see the expected qualitative features emerge neatly in the $M \ll l$ approximation. Refinement of this model should therefore be concentrated on finding a tractable potential that is a good approximation to the vacuum de Sitter potential and could be pieced to the Poschl-Teller potential at the maximum. It should be however remembered here that piecing at the maximum, and resulting matching conditions may result in absence of certain QNMs from the spectrum.

\section{Further investigation of the model}

We have outlined above the steps to be taken for making the QNMs of this model agree well with numerical studies for late time behaviour of the field. However, it must be noted that no analytical approximation proposed so far for time behaviour of fields even in the Schwarzschild black hole reproduces its entire time behaviour from intermediate to late times. The analytical model in 9] reproduces the QNMs, but not the characteristic late time tail for a Schwarzschild perturbation. It is to be noted that our model shows exponential late time decay for the field as seen for the SdS black hole, although the exponent does not match numerical studies. It also shows the QNMs at intermediate times. Therefore it offers an interesting platform to address the following questions:

1) Can one map the complete time behaviour of a perturbation in this model, starting from intermediate times? The incompleteness of the QNMs of this model suggests interesting temporal behaviour when the field switches from a decay governed by the first QNM set to the second. It would be most exciting if in this model, a power law decay were found at these intermediate times. It would then shed light on similar behaviour in the SdS black hole.

2) What happens to the near-extremal limit in this model? The discrepancies of our model QNMs with numerical studies are mainly in the angular mode dependence. We therefore expect that the results of taking the extremal limit in our model (two horizons approaching each other) should be a good approximation to the perturbation behaviour 
when the SdS black hole approaches the Nariai black hole. Investigations of the near-extremal limit for asymptotically flat black holes (Reissner-Nordstrom) show pecularities 22]. Our model could be investigated for such pecularities in the near-extremal limit of the SdS black hole.

It is possible to address the above two questions as this model appears tractable. For reasons elaborated above, such an investigation would indeed be valuable in understanding the response of the SdS black hole to perturbations. This investigation, and further refinements of this model to match numerical studies are the subjects of future work.

\section{ACKNOWLEDGEMENT}

The author would like to thank Eric Poisson for useful comments. This work was supported by a fellowship of the Alexander von Humboldt Foundation.

[1] C.V. Vishveshvara, Nature 227 (1970) 936.

[2] H. Nollert, Class. Quant. Grav. 16 (1999) R159.

[3] K.D. Kokkotas and B.G. Schmidt, Living Rev. Rel. 2 (www.livingreviews.org/Articles/Volume2/1999-2kokkotas).

[4] F. Mellor and I. Moss, Phys. Rev. D41 (1990) 403.

[5] I. Moss and J.P. Norman, Class. Quant. Grav. 19 (2002) 2323.

[6] V. Cardoso and J.P.S. Lemos, gr-qc/0301078

[7] P.R. Brady, C. Chambers, W. Krivan and P. Laguna, Phys. Rev. D55 (1997) 7538.

[8] P.R. Brady, C. Chambers, W. Laarakkers and E. Poisson, Phys. Rev. D 60 (1999) 064003.

[9] V. Ferrari and B. Mashhoon, Phys. Rev. D30 (1984) 295.

[10] J.S.F Chan and R.B. Mann, Phys. Rev. D55 (1997) 7546. QNMs for the BTZ black hole are studied analytically here, but using an approximate potential that reproduces the behaviour of the BTZ potential near the horizon.

[11] T.R. Govindarajan and V. Suneeta, Class. Quant. Grav. 18 (2001) 265.

[12] V. Cardoso and J.P.S. Lemos, Phys. Rev. D63 (2001) 124015; Phys. Rev. D64 (2001) 084017.

[13] G.T. Horowitz and V.E. Hubeny, Phys. Rev. D62 (2000) 024027.

[14] J.S.F. Chan and R.B. Mann, Phys. Rev. D59 (1999) 064025.

[15] B. Wang, E. Abdalla and R.B. Mann, Phys. Rev. D65 (2002) 084006.

[16] R. Aros, C. Martinez, R. Troncoso and J. Zanelli, Phys. Rev. D67, (2003) 044014.

[17] D. Birmingham, I. Sachs and S.N. Solodukhin, Phys. Rev. Lett. 88 (2002) 151301.

[18] D.T. Son and A.O. Starinets, JHEP 0209(2002)042.

[19] A. Strominger, JHEP 0110(2001)034; M. Spradlin, A. Strominger and A. Volovich, Les Houches lectures on deSitter space, hep-th/0110007 and references therein.

[20] E. Abdalla, K.H.C. Castello-Branco and A. Lima-Santos, Phys. Rev. D66 (2002) 104018.

[21] E.S.C. Ching, P.T. Leung, W.M. Suen and K. Young, Phys. Rev. D54 (1996) 3778.

[22] N. Andersson and H. Onozawa, Phys. Rev. D54 (1996) 7470. 